\title{
DESIGN OF INPUT COUPLERS AND ENDCELLS FOR SIDE COUPLED MUFFIN-TIN STRUCTURES
}

\author{
Warner Bruns, TU Berlin, EN-2, Einsteinufer 17, 10587 Berlin
}

\begin{abstract}
The input couplers and endcells for a previously proposed standing wave muf®n-tin structure are designed. Thereby the problem is not only to match the coupler but also to choose the endcells in a way that the coupling cells are not excited. The input coupler has to match the external Q of the structure to the unloaded $\mathrm{Q}$ in order to get zero re-ection. The endcells are designed to re $^{-}$ect the forward travelling wave in such a way, that only the accelerator cells are excited and not the coupling cells. The calculation of the endcell behaviour as well as the calculation of the external Q's are deduced from the knowledge of resonant ${ }^{\circ}$ elds only. The external Q's are calculated with the KrollYu method.
\end{abstract}

\section{INTRODUCTION}

In a previously published paper [1] side coupled muf®n tins were proposed. The structures should operate in a con-uent $\pi$ mode and therefore as standing wave structures. It follows that they only have input couplers and no output couplers. The structures have to be terminated with proper endcells to re-ect the forward travelling wave with the right phase, so that the linear combination of forward and backward travelling wave gives the accelerating standing wave pattern in the main cells.

The input couplers are responsible for achieving a matched load for the power source. For travelling wave structures, an input coupler can be designed by minimizing the re ection of a structure consisting of input coupler - short section of accelerator - output coupler, where the output coupler has the same geometry as the input coupler. The re ection of such a structure can be calculated e.g. with FDTD, assuming a lossless structure.

This does not work for standing wave structures, since they do not have output couplers. Therefore the re ection calculated with e.g. FDTD would ever be one (in magnitude) due to the assumption of no losses. It is quite easy to implement losses in a FDTD-Code, but it is not really necessary for the task of ${ }^{\circledR}$ nding an input coupler for a standing wave structure.

It is known, that the re-ection from a lossy cavity coupled with a waveguide is zero, if the external Q-value due to the coupler matches the internal Q-value due to losses (wall losses and beam loading).

The Q-value due to the wall losses can be calculated by the power loss method, the beam loading can be neglected since we can assume, that more than $90 \%$ of the power will be dissipated in the walls.

How to determine the external Q? Already Slater [2] stated, that the external $\mathrm{Q}$ of a cavity could be calculated (or measured) by moving a totally re ${ }^{-}$ecting plane in the feeding waveguide and observing the frequency shift of the resonance. Kroll and $\mathrm{Yu}$ [3] deduced clever formulas from this principle to calculate the external Q from only 3 frequency/position pairs.
Since these formulas require the knowledge of resonant @elds only, this approach ${ }^{\circledR}$ ts nicely with eigenvalue solvers like $\mathrm{Gd} \circledast \mathrm{dL}[4]$.

\section{DESIGN OF THE ENDCELLS}

The endcells have to re ${ }^{-}$ect the forward travelling wave in the right way to produce a standing wave pattern in the accelerating cells. Since this ®eld is a standing wave, it can be calculated as an eigenvalue problem.

The optimal endcells produce no ${ }^{\circledR}$ eld in the side cavities and -at @eld in the main cavities. Since such a ${ }^{\circledR}$ eld has the highest shunt impedance, the optimal endcell con®guration can be found by maximizing the shunt impedance as a function of the endcell parameters. It was found, that 3 parameters are suf®cient for achieving a ${ }^{-}$at ${ }^{\circledR}$ eld.

An iterative approach was used: maximize the shunt impedance with respect to the ${ }^{\circledR}$ rst parameter (gap of the last main cell) the other two parameters held $® x e d$, then maximize with respect to the second (width of the narrow coupling cell), the other parameters held $₫ x e d$, then maximize with respect to the third. Repeat this procedure three times. It was found that the gap of last main cell should be a factor of 1.1 longer, the width of the last wide coupling cell has to be increased by a factor of 1.2 , and the width of the last narrow coupling cell should be decreased by a factor of 0.67 .

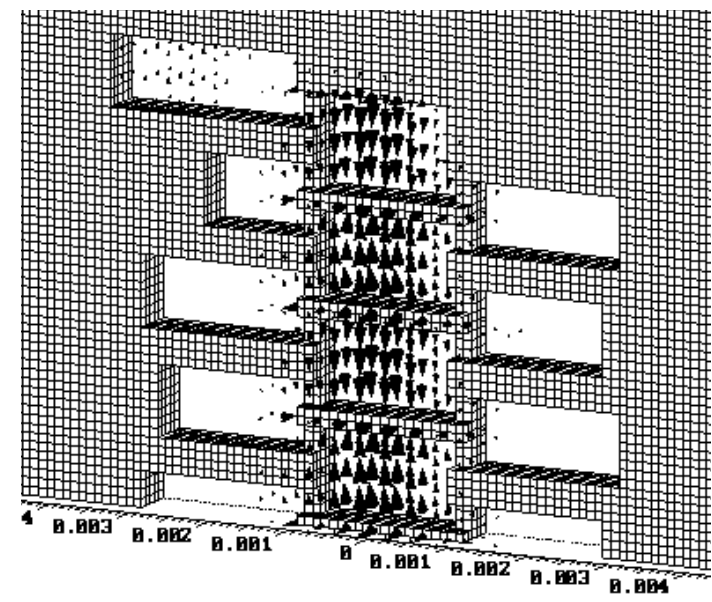

Figure 1. Field near the optimized endcells.

\section{DESIGN OF THE INPUT COUPLER}

I use a -ower paddle coupler. This coupler has two advantages: The used mode in the feeding waveguide is the $T E_{01}$ mode. This mode has a small attenuation that even decreases with growing frequency. The other advantage is, the coupler has to be mechanically attached to only one side of the planar structure. 
The internal Q of the structure is about 2700. The external Q gets calculated with the Kroll-Yu method. To have short computation times I do not calculate the $®$ elds in the total structure, but only a quarter of a section near the coupler. The external Q of the total structure can be determined by scaling.

The external Q is proportional to the stored energy in the structure and inverse proportional to the power ${ }^{-}$ow through the coupler. For a linac, the stored energy is proportional to the length, the power ${ }^{-}$ow is not. Therefore for a given coupler the external $\mathrm{Q}$ grows linearly with the structure length.

Actually I calculated with a quarter of the coupler and $(3+1 / 2)$ half cells of total 56 cells. My stored energy is only $\frac{(3+1 / 2) \frac{1}{2}}{56}$ of the total energy, the power - ow through the coupler is $\frac{1}{4}$ of the total power ${ }^{-}$ow. This means, my calculated $Q$ values are a factor of $\frac{56}{(3+1 / 2) \frac{1}{2}} \frac{1}{4}=8$ lower than if I had modelled the total structure. With an internal Q of 2700, I have to adjust the external Q to $2700 / 8=340$.

Fig. 2 shows the $® e l d$ in the model structure. Fig. 4 shows calculated values for the external $Q$ of my model section. It can be seen, that by varying the $\mathrm{x}$-position of the coupler, a wide range of external Q's can be achieved. Fig. 3 shows the $®$ eld in a structure with half a coupler. The ending position of the groove is choosen to produce a zero of the ®eld at the center of the coupler.

\section{ACKNOWLEDGEMENT}

I would like thank the APS project at Argonne National Laboratory for supporting this work.

\section{References}

[1] H. Henke and W. Bruns, ${ }^{a}$ A Broad-Band Side Coupled mm \pm wave RF structure for relativistic electron acceleration ${ }^{\mathbf{o}}$, Proc. 1993 IEEE Particle Accelerators Conference, Washington DC

[2] J. C. Slater, Microwave Electronics (Van Nostrand, New York, 1950)

[3] N. Kroll and D. Yu, ${ }^{a}$ Computer Determination of the External Q and Resonant Frequency of Waveguide Loaded Cavities $^{\text {', }}$ Particle Accelerators, 1990, Vol. 34, pp. 231-250

[4] W. Bruns, ${ }^{a} \mathrm{Gd} \circledast \mathrm{dL}$ : A Finite Difference Program for Arbitrarily Small Perturbations in Rectangular Geometries ${ }^{\circ}$, to be presented at the COMPUMAG 95, Berlin

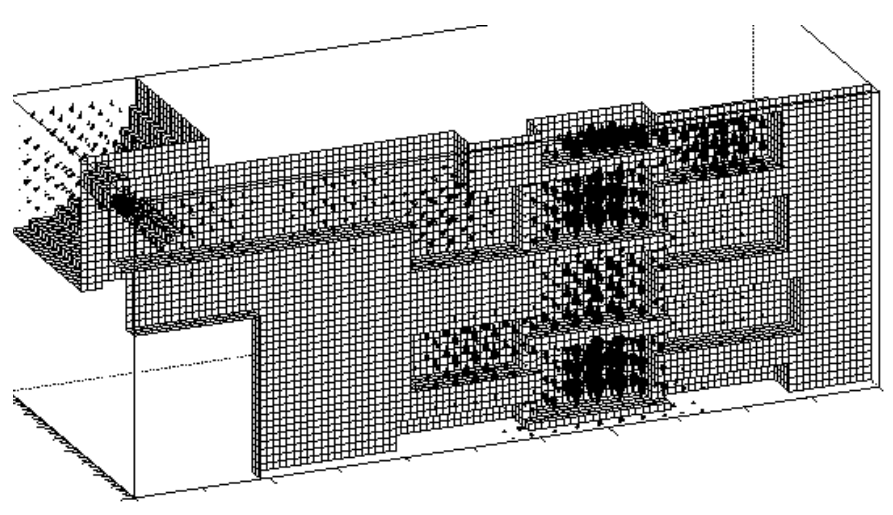

Figure 2. Model which $\mathrm{Q}$ values were computed for. This is a quarter of the coupler and $\frac{(3+1 / 2) \frac{1}{2}}{56}$ of the accelerator. The coupler is at $x=7.1 \mathrm{~mm}$.

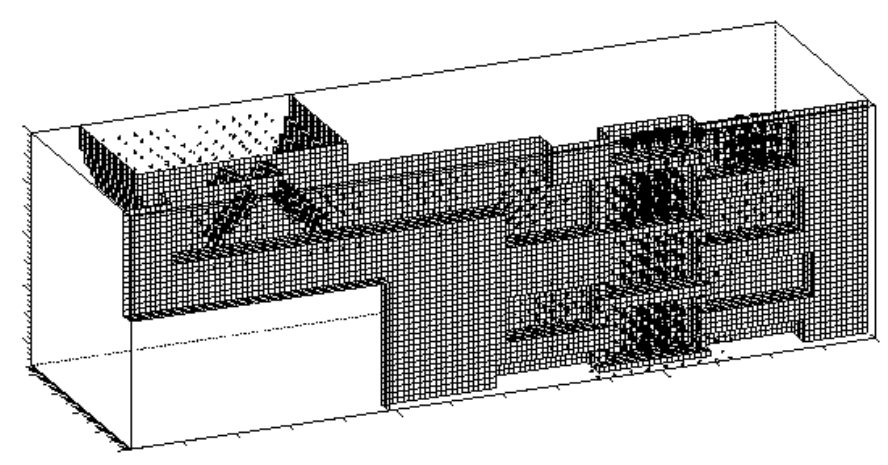

Figure 3. Same structure as above but with a half coupler. Obviously the ®eld is the same in the linac section, but the coupling is twice as high.

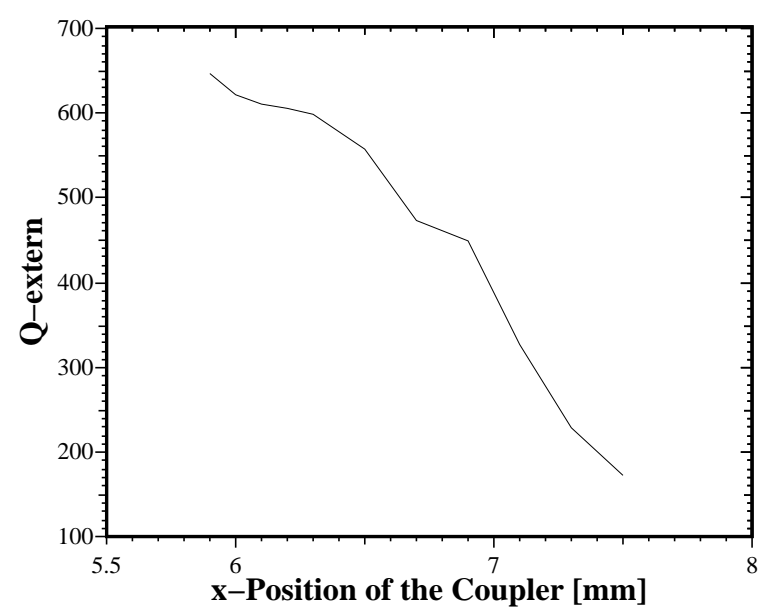

Figure 4. Computed external Q's as a function of the X-Position of the coupler. 